\title{
EXTREMAL EIGENVALUES OF THE CONFORMAL LAPLACIAN UNDER SIRE-XU NORMALIZATION
}

\author{
SAMUEL PÉREZ-AYALA
}

\begin{abstract}
Let $\left(M^{n}, g\right)$ be a closed Riemannian manifold of dimension $n \geq$ 3. We study the variational properties of the $k$-th eigenvalue functional $\tilde{g} \in$ $[g] \mapsto \lambda_{k}\left(L_{\tilde{g}}\right)$ under a non-volume normalization proposed by Sire-Xu. We discuss necessary conditions for the existence of extremal eigenvalues under such normalization. Also, we discuss the general existence problem when $k=$
\end{abstract} 1.

\section{INTRODUCTION}

Let $\left(M^{n}, g\right)$ be a closed (compact, no boundary) Riemannian manifold of dimension $n \geq 3$, equipped with a smooth Riemannian metric $g$. We consider the metric dependent operator known as the Conformal Laplacian defined by

$$
L_{g}:=-\Delta_{g}+c_{n} R_{g} \text {. }
$$

Here $c_{n}=\frac{n-2}{4(n-1)}$ and $\Delta_{g}$ is the Laplace-Beltrami operator defined as a negative operator. $L_{g}$ is conformally covariant in the following sense: if $\tilde{g}=\mu^{4 /(n-2)} g$, then for any $u \in C^{\infty}\left(M^{n}\right)$ we have

$$
L_{\mu^{\frac{4}{n-2}} g}(u)=\mu^{-\frac{n+2}{n-2}} L_{g}(\mu u) .
$$

Since $M^{n}$ is compact, $L_{g}$ has a discrete spectrum and we denote it by

$$
\lambda_{1}\left(L_{g}\right)<\lambda_{2}\left(L_{g}\right) \leq \lambda_{3}\left(L_{g}\right) \leq \cdots \leq \lambda_{k}\left(L_{g}\right) \rightarrow \infty,
$$

where each eigenvalue is repeated according to their multiplicity. Notice that the first eigenvalue is always simple.

There are many conformally invariant quantities associated with $L_{g}$. First, the sign of $\lambda_{1}\left(L_{g}\right)$ is conformally invariant. Indeed, the sign of the Yamabe invariant $Y\left(M^{n},[g]\right)$,

$$
Y\left(M^{n},[g]\right):=\inf _{u \in W^{1,2}\left(M^{n}, g\right)} \frac{\int_{M} u L_{g} u d v_{g}}{\left(\int_{M}|u|^{\frac{2 n}{n-2}} d v_{g}\right)^{\frac{n-2}{n}}},
$$

coincides with the sign of $\lambda_{1}\left(L_{g}\right)$ ([8]). Secondly, the dimension of $\operatorname{Ker}\left(L_{g}\right)$ is also conformally invariant. This follows from the transformation law (1.2).

In this paper we will investigate the variational properties of the eigenvalue functional

$$
\tilde{g} \in[g] \longmapsto \lambda_{k}\left(L_{\tilde{g}}\right)
$$

under a non-standard normalization proposed by Sire-Xu in 13. Notice that for any positive real number $c>0$, the $k$-th eigenvalue scales as $\lambda_{k}\left(L_{c g}\right)=c^{-1} \lambda_{k}\left(L_{g}\right)$. 
This can be observed from (1.2). Therefore, in order to study possible "critical points" of this functional, some sort of normalization is required. The most geometrically natural and standard normalization is done by restricting the $k$-th eigenvalue functional (1.5) to conformal metrics with fixed volume. The study of this functional under such volume constraint is equivalent to study

$$
\tilde{g} \in[g] \longmapsto \lambda_{k}\left(L_{\tilde{g}}\right) \operatorname{Vol}\left(M^{n}, \tilde{g}\right)^{\frac{2}{n}} .
$$

If one writes $\tilde{g}=\mu^{4 /(n-2)} g$, this translates to

$$
\tilde{g} \in[g] \longmapsto \lambda_{k}\left(L_{\tilde{g}}\right)\left(\int_{M} \mu^{\frac{2 n}{n-2}} d v_{g}\right)^{\frac{2}{n}}
$$

For the positive part of the spectrum of $L_{g}$, Ammann-Jammes proved that the volume normalization is not suitable if one is looking for Riemannian metrics achieving the supremum of the $k$-th eigenvalue functional in conformal classes ([1). Specifically, if $\lambda_{+}\left(L_{g}\right)$ is the first positive eigenvalue for $L_{g}$, then

$$
\Lambda_{+}\left(M^{n},[g]\right):=\sup _{\tilde{g} \in[g]} \lambda_{+}\left(L_{\tilde{g}}\right) \operatorname{Vol}(M, \tilde{g})^{\frac{2}{n}}=\infty .
$$

For instance, if $n \geq 3$, then $\sup _{\tilde{g} \in\left[g_{r}\right]} \lambda_{1}\left(L_{\tilde{g}}\right) \operatorname{Vol}\left(\mathbb{S}^{n}, \tilde{g}\right)^{2 / n}=\infty$, where $g_{r}$ is the round metric. This is part of a much more general phenomenon: if $A_{g}$ is a conformally covariant elliptic operator whose order is less than the dimension of the manifold, and if $A_{g}$ is invertible on the cylinder $\mathbb{S}^{n-1} \times \mathbb{R}$, then

$$
\bar{\Lambda}_{+}\left(M^{n},[g]\right):=\sup _{\tilde{g} \in[g]} \lambda_{+}\left(A_{\tilde{g}}\right) \operatorname{Vol}(M, \tilde{g})^{\frac{2}{n}}=\infty,
$$

where $\lambda_{+}\left(A_{g}\right)$ denotes the first positive eigenvalue of $A_{g}([1])$. This puts a restriction on the type of operators for which one can try to find extremal eigenvalues by maximizing over conformal classes.

The main idea by Sire-Xu in [13] was to show that there is another way to normalize (1.5) such that the supremum over conformal metrics is finite, even for the positive part of spectrum of $L_{g}$. Let us define $F_{g}^{k}$ by

$$
\tilde{g}=\mu^{\frac{4}{n-2}} g \in[g] \longmapsto F_{g}^{k}(\tilde{g}):=\lambda_{k}\left(L_{\tilde{g}}\right) \int_{M} \mu^{\frac{4}{n-2}} d v_{g} .
$$

Sire-Xu showed that for any metric $g$ on $M^{n}$, the quantity

$$
\Lambda_{k}\left(M^{n}, g\right):=\sup _{\tilde{g}=\mu^{\frac{4}{n-2}}} \lambda_{g \in[g]}\left(L_{\tilde{g}}\right) \int_{M} \mu^{\frac{4}{n-2}} d v_{g},
$$

is finite (see Section 6 in [13]). Unlike previously considered quantities, $\Lambda_{k}\left(M^{n}, g\right)$ is not conformally invariant, i.e. it depends on the choice of the metric $g$. However, questions regarding the existence of maximal metrics for (1.11), their regularity, and its geometric meaning could still be asked.

In the case of the $k$-th eigenvalue functional under volume normalization, (1.7), harmonic maps have been found to be in connection with maximal metrics. Let $[g]$ be a conformal class for which $L_{g}$ has at least two negative eigenvalues and trivial kernel. Gursky and the author proved in [6] that to each generalized metric $\tilde{g}=\mu^{4 /(n-2)} g$ (see Section 2 in [6] for definition) maximizing (1.7) with $k=2$, there is a collection of $C^{2, \alpha}$-functions $\left\{u_{1}, \cdots, u_{p}\right\}$, each of them solving $L_{g}\left(u_{i}\right)=$ $\lambda_{2}\left(L_{\tilde{g}}\right) u_{i} \mu^{4 /(n-2)}$, such that $\sum_{i=1}^{p} u_{i}^{2}=\mu^{2}$. If $p>1$, then $\left(M^{n} \backslash\{\mu=0\}, \tilde{g}\right)$ admits a harmonic map into a sphere. Our first result is a step in the same direction: 
Theorem 1.1. Let $\left(M^{n}, g\right)$ be a closed Riemannian manifold of dimension $n \geq 3$ and with a unit volume metric $g$. Let $g_{e}=\mu_{e}^{4 /(n-2)} \mathrm{g}$ be a smooth extremal metric for $F_{g}^{k}$ (see Definition [3.2) with $\int_{M} \mu_{e}^{4 /(n-2)} d v_{g}=1$. If either

$$
\lambda_{k}\left(L_{g_{e}}\right)>\lambda_{k-1}\left(L_{g_{e}}\right) \text { or } \quad \lambda_{k}\left(L_{g_{e}}\right)<\lambda_{k+1}\left(L_{g_{e}}\right),
$$

and $\lambda_{k}\left(L_{g_{e}}\right) \neq 0$, then there exists a finite family $\left\{u_{1}, \cdots, u_{p}\right\}$ of eigenfunctions associated to $\lambda_{k}\left(L_{g_{e}}\right)$ such that $\sum_{i=1}^{p} u_{i}^{2} \mu_{e}^{2} \equiv 1$. In particular, the map $U=$ $\left(u_{1} \mu_{e}, \cdots, u_{p} \mu_{e}\right): M \rightarrow \mathbb{S}^{p-1}$ is well defined. As a consequence,

$$
\lambda_{k}\left(L_{g_{e}}\right)=-\frac{1}{2} \mu_{e}^{2} \Delta_{g_{e}}\left(\mu_{e}^{-2}\right)+\mu_{e}^{2} \sum_{i=1}^{p}\left|\nabla_{g_{e}} u_{i}\right|_{g_{e}}^{2}+c_{n} R_{g_{e}} .
$$

Furthermore, if the extremal metric $g_{e}$ coincides with the background metric g, i.e. if $\mu_{e}=1$, then $\left(M^{n}, g_{e}\right)$ admits a harmonic map into a sphere.

Notice that for any metric $\tilde{g} \in[g]$, the first eigenvalue $\lambda_{1}\left(L_{\tilde{g}}\right)$ always satisfies the second condition in (1.12). As a consequence of Theorem 1.1 we derive a necessary condition for the existence of extremal metrics for $F_{g}^{1}$ : the sign of $R_{g}$ and $Y\left(M^{n},[g]\right)$ need to coincide. Corollary 1.2 discusses the result in the case when $Y\left(M^{n},[g]\right)>0$, but the same holds in the case of negative Yamabe invariant; see Corollary 3.4.

Corollary 1.2. Suppose $[g]$ is a conformal class for which $Y\left(M^{n},[g]\right)>0$, and assume that $g_{e}=\mu_{e}^{4 /(n-2)} g$ is an extremal metric for $F_{g}^{1}$ with $\int_{M} \mu_{e}^{4 /(n-2)} d v_{g}=1$. Then

$$
c_{n} R_{g}=\lambda_{1}\left(L_{g_{e}}\right) \mu_{e}^{\frac{4}{n-2}} .
$$

In particular, the scalar curvature $R_{g}$ with respect to the background metric $g$ is positive everywhere.

We also solve the existence problem of maximal metrics for the first eigenvalue functional under Sire-Xu normalization, i.e. we solve the maximization problem for $F_{g}^{1}$. In fact, the condition of both $R_{g}$ and $Y\left(M^{n},[g]\right)$ having the same sign is also sufficient. Theorem 1.3 discusses the case when $Y\left(M^{n},[g]\right)>0$, but the same conclusions hold in the case of negative Yamabe invariant; see Theorem 3.5.

Theorem 1.3. Let $M$ be a closed n-dimensional manifold endowed with a conformal class [g] satisfying $Y(M,[g])>0$. If $R_{g}>0$, then the metric

$$
g_{\max }:=\mu_{\max }^{\frac{4}{n-2}} g=\left(\frac{R_{g}}{\int_{M} R_{g} d v_{g}}\right) g
$$

solves the maximization problem for $\Lambda_{1}(M, g)$, i.e. $F_{g}^{1}\left(g_{\max }\right)=\Lambda_{1}(M, g)$.

Theorems 1.3 and 3.5 provide a generalization of the results found in [13, where the authors showed that the round metric was the unique maximizer for $F_{g_{r}}^{1}$ on $\mathbb{S}^{n}$. With these results the existence problem for $k=1$ is fully understood. The existence theory for higher eigenvalues is still unknown.

The paper is organized as follows. In Section 2 we recall an important result from classical analytic perturbation theory. In particular, we discuss a result due to Canzani (3]) which is the base for this work. The techniques employed to prove Theorem 2.2 follow closely those used by Soufi-Ilias in 4. In Section 3 is where we proved our main results, including those analogous to Corollary 1.2 and Theorem 
1.3 in the case when $Y\left(M^{n},[g]\right)<0$. The proof of Theorem 1.1 uses classical separation theorems as it was done in [4], 5].

\section{Background On Analytic Perturbation Theory}

We will introduce a more general notion than that of maximal metrics. Loosely speaking, extremal metrics will be those which are "critical points" of the functional $\lambda_{k}(L$. $)$ under the Sire-Xu normalization. As we shall discuss, maximal metrics are always extremal, but the converse may not be true. To formally define what extremal metrics are, we need first to show the existence of the one-sided derivatives of $\lambda_{k}(L$.$) along conformal analytic perturbations.$

To this end, let $g(t)=\mu_{t}^{4 /(n-2)} \tilde{g}$ be any analytic deformation of $\tilde{g} \in[g]$, i.e. $\mu_{0} \equiv 1$ and $\mu_{t}$ depends real analytically in $t$. It is good to note that we are only considering deformations that stay in a given conformal class. The function $\mu_{t}$ is called the generating function for the deformation $g(t)$. In general, the eigenvalue functional $\lambda_{k}\left(L_{g(t)}\right)$ is continuous, but not differentiable. However, and as we mentioned before, both $\left.\frac{d}{d t} \lambda_{k}\left(L_{g(t)}\right)\right|_{t=0^{+}}$and $\left.\frac{d}{d t} \lambda_{k}\left(L_{g(t)}\right)\right|_{t=0^{-}}$exist. The existence of the one-sided derivatives relies on the following theorem from perturbation theory for linear operators. The original theory traces back to Rellich-Kato's work, but it was Canzani in 3 who proved that such a theory could be applied to a certain class of conformally covariant operators acting on smooth bundles over $M^{n}$; see also [2].

Theorem 2.1 (Rellich-Kato, Canzani). Let $\lambda_{k}\left(L_{\tilde{g}}\right)$ be the $k$-th eigenvalue of the Conformal Laplacian with respect to $\tilde{g} \in[g]$, and denote by $m$ its multiplicity. Pick any analytic deformation $g(t)=\mu_{t}^{4 /(n-2)} \tilde{g}$ of $\tilde{g}$. Then there exist $\Lambda_{1}(t), \cdots, \Lambda_{m}(t)$ analytic in $t$, and $u_{1}(t), \cdots, u_{m}(t)$ convergent power series in $t$ with respect to the $L^{2}$ norm topology, such that

$$
L_{g(t)} u_{i}(t)=\Lambda_{i}(t) u_{i}(t) \text {, with } \Lambda_{i}(0)=\lambda_{k}\left(L_{\tilde{g}}\right) \text { for all } i=1, \cdots, m ;
$$

and

$$
\int_{M} u_{i}(t) u_{j}(t) d v_{g(t)}=\delta_{i j} \text { for all } i=1, \cdots, m .
$$

Moreover, if we select positive constants $d_{1}$ and $d_{2}$ such that the spectrum of $L_{\tilde{g}}$ in $\left(\lambda_{k}\left(L_{\tilde{g}}\right)-d_{1}, \lambda_{k}\left(L_{\tilde{g}}\right)+d_{2}\right)$ consists only of $\lambda_{k}\left(L_{\tilde{g}}\right)$, then one can find a small enough $\delta>0$ such that the spectrum of $L_{g(t)}$ in the same interval $\left(\lambda_{k}\left(L_{\tilde{g}}\right)-d_{1}, \lambda_{k}\left(L_{\tilde{g}}\right)+d_{2}\right)$ consists of $\left\{\Lambda_{1}(t), \cdots, \Lambda_{m}(t)\right\}$ alone for $|t|<\delta$.

We find it important to notice what the theorem does not assert. For a conformal metric $\tilde{g} \in[g]$, given an eigenvalue $\lambda_{k}\left(L_{\tilde{g}}\right)$ with multiplicity $m$ and an orthonormal basis $\left\{u_{i}\right\}_{i=1}^{m}$ of the $k$-th eigenspace $E_{k}\left(L_{\tilde{g}}\right)$, Theorem 2.1 does not provide a way to find a perturbation $g(t)=\mu_{t}^{4 /(n-2)} \tilde{g}$ of $\tilde{g}$ with a collection of convergent power series $\left\{u_{i}(t)\right\}_{i=1}^{m}$ satisfying (2.1) and (2.2) such that $u_{i}(0)=u_{i}$. In fewer words, orthonormal bases of $E_{k}\left(L_{\tilde{g}}\right)$ cannot be prescribed.

A crucial consequence of Theorem 2.1 is:

Theorem 2.2. Let $g(t)=\mu_{t}^{4 /(n-2)} \tilde{g}$ be any analytic deformation of $\tilde{g}$, i.e. $g(t)$ is analytic with respect to $t$ in a neighborhood of $t=0$, and $g(0)=\tilde{g}\left(\mu_{0} \equiv 1\right)$. Then the one-sided derivatives of $\lambda_{k}\left(L_{g(t)}\right)$,

$$
\left.\frac{d}{d t} \lambda_{k}\left(L_{g(t)}\right)\right|_{t=0^{+}} \text {and }\left.\frac{d}{d t} \lambda_{k}\left(L_{g(t)}\right)\right|_{t=0^{-}}
$$


both exist. Moreover, an explicit formula can be computed in certain cases:

(i) If $\lambda_{k}\left(L_{\tilde{g}}\right)>\lambda_{k-1}\left(L_{\tilde{g}}\right)$, then

$$
\left.\frac{d}{d t} \lambda_{k}\left(L_{g(t)}\right)\right|_{t=0^{+}}=\min _{1 \leq i \leq m} \Lambda_{i}^{\prime}(0)
$$

and

$$
\left.\frac{d}{d t} \lambda_{k}\left(L_{g(t)}\right)\right|_{t=0^{-}}=\max _{1 \leq i \leq m} \Lambda_{i}^{\prime}(0) .
$$

(ii) If $\lambda_{k}\left(L_{\tilde{g}}\right)<\lambda_{k+1}\left(L_{\tilde{g}}\right)$, then

$$
\left.\frac{d}{d t} \lambda_{k}\left(L_{g(t)}\right)\right|_{t=0^{+}}=\max _{1 \leq i \leq m} \Lambda_{i}^{\prime}(0)
$$

and

$$
\left.\frac{d}{d t} \lambda_{k}\left(L_{g(t)}\right)\right|_{t=0^{-}}=\min _{1 \leq i \leq m} \Lambda_{i}^{\prime}(0) .
$$

In both cases,

$$
\Lambda_{i}^{\prime}(0)=-\frac{4}{n-2} \lambda_{k}\left(L_{\tilde{g}}\right) \int_{M} h u_{i}^{2} d v_{\tilde{g}}
$$

Here the notation is as in Theorem [2.1, and $h$ denotes $\left.\frac{d}{d t} \mu_{t}\right|_{t=0}$.

Notice that if we know the sign of $\lambda_{k}\left(L_{g}\right)$, then the one-sided derivatives can be rewritten in terms of formula (2.7). For instance, if $\lambda_{k}\left(L_{\tilde{g}}\right)>0$ and $\lambda_{k}\left(L_{\tilde{g}}\right)>$ $\lambda_{k-1}\left(L_{\tilde{g}}\right)$, so that we are in case (i), then

$$
\left.\frac{d}{d t} \lambda_{k}\left(L_{g(t)}\right)\right|_{t=0^{+}}=-\frac{4}{n-2} \lambda_{k}\left(L_{\tilde{g}}\right) \max _{1 \leq i \leq m} \int_{M} h u_{i}^{2} d v_{\tilde{g}}
$$

and

$$
\left.\frac{d}{d t} \lambda_{k}\left(L_{g(t)}\right)\right|_{t=0^{-}}=-\frac{4}{n-2} \lambda_{k}\left(L_{\tilde{g}}\right) \min _{1 \leq i \leq m} \int_{M} h u_{i}^{2} d v_{\tilde{g}}
$$

Proof. Let us start by pointing out that the family $\left\{\Lambda_{1}(t), \cdots, \Lambda_{m}(t)\right\}$ is an unordered family of eigenvalues of $L_{g(t)}$. From Theorem 2.1 in some neighborhood of $t=0$, the spectrum of $L_{g(t)}$ consists only of $\left\{\Lambda_{1}(t), \cdots, \Lambda_{m}(t)\right\}$. The continuity of $t \mapsto \lambda_{k}\left(L_{g(t)}\right)$ and the analyticity of $t \mapsto \Lambda_{i}(t)$ in a neighborhood of $t=0$ implies that there exists $a, b \in\{1, \cdots, m\}$ such that

$$
\lambda_{k}\left(L_{g(t)}\right)= \begin{cases}\Lambda_{a}(t) & \text { if } t \in[0, \eta) \\ \Lambda_{b}(t) & \text { if } t \in(-\eta, 0] .\end{cases}
$$

for some $\eta>0$. This shows the existence of the one-sided derivatives of $\lambda_{k}\left(L_{g(t)}\right)$ at $t=0$.

Assume now that $\lambda_{k}\left(L_{\tilde{g}}\right)>\lambda_{k-1}\left(L_{\tilde{g}}\right)$. By choosing $d>0$ such that $\lambda_{k-1}\left(L_{\tilde{g}}\right) \notin$ $\left(\lambda_{k}\left(L_{\tilde{g}}\right)-d, \lambda_{k}\left(L_{\tilde{g}}\right)+d\right)$, thanks to Theorem 2.1 one can select a small enough $\eta>\delta>0$ such that $\operatorname{Spect}\left(L_{g(t)}\right) \cap\left(\lambda_{k}\left(L_{\tilde{g}}\right)-d, \lambda_{k}\left(L_{\tilde{g}}\right)+d\right)=\left\{\Lambda_{1}(t), \cdots, \Lambda_{m}(t)\right\}$ for $|t|<\delta$. This together with the continuity of $\lambda_{k-1}\left(L_{g(t)}\right)$ implies that $\Lambda_{i}(t)>$ $\lambda_{k-1}\left(L_{g(t)}\right)$. Therefore, $\Lambda_{i}(t) \geq \lambda_{k}\left(L_{g(t)}\right)$ and thus $\lambda_{k}\left(L_{g(t)}\right)=\min _{1 \leq i \leq m}\left\{\Lambda_{i}(t)\right\}$ for $|t|<\delta$. 
We now use (2.10) to deduce $\lambda_{k}\left(L_{g(t)}\right)=\Lambda_{a}(t) \leq \Lambda_{i}(t)$ for all $i=1, \cdots, m$ and $t \in[0, \delta)$, and $\lambda_{k}\left(L_{g(t)}\right)=\Lambda_{b}(t) \leq \Lambda_{i}(t)$ for all $i=1, \cdots, m$ and $t \in(-\delta, 0]$. Using $\lambda_{k}\left(L_{g(t)}\right)=\Lambda_{a}(t) \leq \Lambda_{i}(t)$ with $t$ and $i$ as specified, one obtains

$$
\frac{\Lambda_{a}(t)-\lambda_{k}\left(L_{\tilde{g}}\right)}{t} \leq \frac{\Lambda_{i}(t)-\lambda_{k}\left(L_{\tilde{g}}\right)}{t} \text { for } t \in(0, \delta) \Rightarrow \Lambda_{a}^{\prime}(0) \leq \Lambda_{i}^{\prime}(0) .
$$

Similarly, from $\lambda_{k}\left(L_{g(t)}\right)=\Lambda_{b}(t) \leq \Lambda_{i}(t)$, we deduce

$$
\frac{\Lambda_{b}(t)-\lambda_{k}\left(L_{\tilde{g}}\right)}{t} \geq \frac{\Lambda_{i}(t)-\lambda_{k}\left(L_{\tilde{g}}\right)}{t} \text { for } t \in(-\delta, 0) \Rightarrow \Lambda_{b}^{\prime}(0) \geq \Lambda_{i}^{\prime}(0) .
$$

Putting all together we get

$$
\left.\frac{d}{d t} \lambda_{k}\left(L_{g(t)}\right)\right|_{t=0^{+}}=\min _{1 \leq i \leq m} \Lambda_{i}^{\prime}(0) \text { and }\left.\frac{d}{d t} \lambda_{k}\left(L_{g(t)}\right)\right|_{t=0^{-}}=\max _{1 \leq i \leq m} \Lambda_{i}^{\prime}(0),
$$

which is what we wanted to show. If $\lambda_{k}\left(L_{\tilde{g}}\right)<\lambda_{k+1}\left(L_{\tilde{g}}\right)$, then a similar analysis leads to $\lambda_{k}\left(L_{g(t)}\right)=\max _{1 \leq i \leq m}\left\{\Lambda_{i}(t)\right\}$. The proof for this case is similar and it is therefore omitted.

It remains to show (2.7). We use the eigenvalue equation (2.1), together with the transformation law (1.2):

$$
L_{\tilde{g}}\left(\mu_{t} u_{i}(t)\right)=\Lambda_{i}(t) \mu_{t}^{\frac{n+2}{n-2}} u_{i}(t) .
$$

Differentiating this equation with respect to $t$ and then setting $t=0$ gives us

$$
L_{\tilde{g}}\left(h u_{i}\right)+L_{\tilde{g}}\left(u_{i}^{\prime}\right)=\Lambda_{i}^{\prime}(0) u_{i}+\frac{n+2}{n-2} \lambda_{k}\left(L_{\tilde{g}}\right) h u_{i}+\lambda_{k}\left(L_{\tilde{g}}\right) u_{i}^{\prime},
$$

where $u_{i}=u_{i}(0)$ and $u_{i}^{\prime}=\left.\frac{d}{d t} u_{i}(t)\right|_{t=0}$. Also, by setting $t=0$ in (2.1) one obtains $L_{\tilde{g}} u_{i}=\lambda_{k}\left(L_{\tilde{g}}\right) u_{i}$, and multiplying this by $u_{i}^{\prime}$ gives us

$$
u_{i}^{\prime} L_{\tilde{g}} u_{i}=\lambda_{k}\left(L_{\tilde{g}}\right) u_{i}^{\prime} u_{i}
$$

On the other hand, one can multiply (2.15) by $u_{i}$ to get

$$
u_{i} L_{\tilde{g}}\left(h u_{i}\right)+u_{i} L_{\tilde{g}} u_{i}^{\prime}=\Lambda_{i}^{\prime}(0) u_{i}^{2}+\frac{n+2}{n-2} \lambda_{k}\left(L_{\tilde{g}}\right) h u_{i}^{2}+\lambda_{k}\left(L_{\tilde{g}}\right) u_{i} u_{i}^{\prime} .
$$

After subtracting (2.16) from (2.17), and integrating with respect to $d v_{\tilde{g}}$ we obtain

$$
\begin{aligned}
\int_{M} u_{i} L_{\tilde{g}}\left(h u_{i}\right) d v_{\tilde{g}}+\underbrace{\int_{M}\left(u_{i} L_{\tilde{g}} u_{i}^{\prime}-u_{i}^{\prime} L_{\tilde{g}} u_{i}\right) d v_{\tilde{g}}}_{=0 \text { by self-adjointness of } L_{\tilde{g}}} & =\Lambda_{i}^{\prime}(0) \underbrace{\int_{M} u_{i}^{2} d v_{\tilde{g}}}_{=1 \text { by }(1.2)} \\
& +\frac{n+2}{n-2} \lambda_{k}\left(L_{\tilde{g}}\right) \int_{M} h u_{i}^{2} d v_{\tilde{g}} \\
\int_{M} L_{\tilde{g}}\left(u_{i}\right) \cdot\left(h u_{i}\right) d v_{\tilde{g}} & =\Lambda_{i}^{\prime}(0)+\frac{n+2}{n-2} \lambda_{k}\left(L_{\tilde{g}}\right) \int_{M} h u_{i}^{2} d v_{\tilde{g}} \\
\lambda_{k}\left(L_{\tilde{g}}\right) \int_{M} h u_{i}^{2} d v_{\tilde{g}} & =\Lambda_{i}^{\prime}(0)+\frac{n+2}{n-2} \lambda_{k}\left(L_{\tilde{g}}\right) \int_{M} h u_{i}^{2} d v_{\tilde{g}} .
\end{aligned}
$$

Hence

$$
\Lambda_{i}^{\prime}(0)=-\frac{4}{n-2} \lambda_{k}\left(L_{\tilde{g}}\right) \int_{M} h u_{i}^{2} d v_{\tilde{g}}
$$

This concludes the proof of $(i)$. The arguments for $(i i)$ are similar and therefore we omit them. 


\section{Extremal Metrics under Sire-Xu Normalization}

We start with Corollary 3.1, which is an immediate consequence of Theorem 2.2 ,

Corollary 3.1. Let $g(t)=\mu_{t}^{4 /(n-2)} \tilde{g}=\mu_{t}^{4 /(n-2)} \tilde{\mu}^{4 /(n-2)} g$ be any analytic deformation of $\tilde{g} \in[g]$. Then the one-sided derivatives of $F_{g}^{k}(g(t))$ both exist. Let us further assume that the conformal factor of the perturbed metric satisfies

$$
\int_{M} \tilde{\mu}^{\frac{4}{n-2}} d v_{g}=1
$$

Then an explicit formula for the one-sided derivatives can be computed in certain cases:

(i) if $\lambda_{k}\left(L_{\tilde{g}}\right)>\lambda_{k-1}\left(L_{\tilde{g}}\right)$, then

$$
\left.\frac{d}{d t} F_{g}^{k}(g(t))\right|_{t=0^{+}}=\frac{4}{n-2} \lambda_{k}\left(L_{\tilde{g}}\right) \int_{M} h \tilde{\mu}^{\frac{4}{n-2}} d v_{g}+\min _{1 \leq i \leq m} \Lambda_{i}^{\prime}(0)
$$

and

$$
\left.\frac{d}{d t} F_{g}^{k}(g(t))\right|_{t=0^{-}}=\frac{4}{n-2} \lambda_{k}\left(L_{\tilde{g}}\right) \int_{M} h \tilde{\mu}^{\frac{4}{n-2}} d v_{g}+\max _{1 \leq i \leq m} \Lambda_{i}^{\prime}(0)
$$

If we select as generating function $\mu_{t}=e^{t w \tilde{\mu}^{2}}$, where $w$ has zero mean value with respect to $\tilde{g}$, then

$$
\left.\frac{d}{d t} F_{g}^{k}(g(t))\right|_{t=0^{+}}=\min _{1 \leq i \leq m} \Lambda_{i}^{\prime}(0),
$$

and

$$
\left.\frac{d}{d t} F_{g}^{k}(g(t))\right|_{t=0^{-}}=\max _{1 \leq i \leq m} \Lambda_{i}^{\prime}(0) .
$$

(ii) if $\lambda_{k}\left(L_{\tilde{g}}\right)<\lambda_{k+1}\left(L_{\tilde{g}}\right)$, then

$$
\begin{aligned}
& \left.\frac{d}{d t} F_{g}^{k}(g(t))\right|_{t=0^{+}}=\frac{4}{n-2} \lambda_{k}\left(L_{\tilde{g}}\right) \int_{M} h \tilde{\mu}^{\frac{4}{n-2}} d v_{g}+\max _{1 \leq i \leq m} \Lambda_{i}^{\prime}(0), \\
& \text { and } \begin{aligned}
\left.\frac{d}{d t} F_{g}^{k}(g(t))\right|_{t=0^{-}} & =\frac{4}{n-2} \lambda_{k}\left(L_{\tilde{g}}\right) \int_{M} h \tilde{\mu}^{\frac{4}{n-2}} d v_{g}+\min _{1 \leq i \leq m} \Lambda_{i}^{\prime}(0) .
\end{aligned}
\end{aligned}
$$

If we select as generating function $\mu_{t}=e^{t w \tilde{\mu}^{2}}$, where $w \in L^{2}(M, \tilde{g})$ has zero mean value with respect to $\tilde{g}$, then

$$
\left.\frac{d}{d t} F_{g}^{k}(g(t))\right|_{t=0^{+}}=\max _{1 \leq i \leq m} \Lambda_{i}^{\prime}(0)
$$

and

$$
\left.\frac{d}{d t} F_{g}^{k}(g(t))\right|_{t=0^{-}}=\min _{1 \leq i \leq m} \Lambda_{i}^{\prime}(0)
$$

Here the notation is as in Theorem 2.2.

Proof. The proof follows directly from Theorem 2.2. Using

$$
F_{g}^{k}(g(t))=\lambda_{k}\left(L_{g(t)}\right) \int_{M} \mu_{t}^{\frac{4}{n-2}} \cdot \mu^{\frac{4}{n-2}} d v_{g}
$$

we see that the one-sided derivatives of $F_{g}^{k}(g(t))$ both exist. Assuming $\lambda_{k}\left(L_{\tilde{g}}\right)>$ $\lambda_{k-1}\left(L_{\tilde{g}}\right)$, we compute $\left.\frac{d}{d t} F_{g}^{k}(g(t))\right|_{t=0^{+}}$as follows: 


$$
\begin{aligned}
\left.\frac{d}{d t} F_{g}^{k}(g(t))\right|_{t=0^{+}}= & \left.\frac{d}{d t} \lambda_{k}\left(L_{g(t)}\right)\right|_{t=0^{+}} \cdot \int_{M} \mu_{0}^{\frac{4}{n-2}} \tilde{\mu}^{\frac{4}{n-2}} d v_{g} \\
& +\frac{4}{n-2} \lambda_{k}\left(L_{\tilde{g}}\right) \int_{M} \mu_{0}^{\frac{6-n}{n-2}} \tilde{\mu}^{\frac{4}{n-2}} h d v_{g} \\
= & \min _{1 \leq i \leq m} \Lambda_{i}^{\prime}(0) \cdot \underbrace{\int_{M} \tilde{\mu}^{\frac{4}{n-2}} d v_{g}}_{=1 \text { by assumption on } \tilde{\mu}}+\frac{4}{n-2} \lambda_{k}\left(L_{\tilde{g}}\right) \int_{M} h \tilde{\mu}^{\frac{4}{n-2}} d v_{g},
\end{aligned}
$$

which is the desired expression. The computations for $\left.\frac{d}{d t} F_{g}^{k}(g(t))\right|_{t=0^{-}}$, as well as for the case $\lambda_{k}\left(L_{\tilde{g}}\right)<\lambda_{k+1}\left(L_{\tilde{g}}\right)$, are similar and are therefore omitted. This concludes the proof.

The previous result allows us to finally define what an extremal metric is.

Definition 3.2. The metric $\tilde{g} \in[g]$ is said to be extremal for the functional $F_{g}^{k}$ if for any deformation $g(t)$ of $\tilde{g}$ which is analytic in a neighborhood of $t=0$, we have

$$
\left.\left.\frac{d}{d t} F_{g}^{k}(g(t))\right|_{t=0^{+}} \cdot \frac{d}{d t} F_{g}^{k}(g(t))\right|_{t=0^{-}} \leq 0
$$

These extremal metrics do not only catch global maximums, but also local extremums, including minimums. How to distinguish these different types of extremal metrics is another important question. Note that solving the maximization problem for $\Lambda_{k}(M, g)$ is equivalent to finding $g_{\max } \in[g]$ such that $F_{g}^{k}\left(g_{\max }\right)=\Lambda_{k}(M, g)$. In particular, any maximal metric is extremal.

In Section 1, we discuss how in certain cases the existence of maximal metrics for (1.7) is associated with existence of harmonic maps into spheres. The connection between extremal eigenvalues and harmonic maps was found first in the case of eigenvalues for the Laplace-Beltrami operator under volume normalization. Here, if $g_{e} \in[g]$ is an extremal metric (defined analogously) for $\tilde{g} \in[g] \mapsto$ $\lambda_{k}\left(-\Delta_{\tilde{g}}\right) \operatorname{Vol}\left(M^{n}, \tilde{g}\right)^{2 / n}$, then the Riemannian manifold $\left(M^{n}, g_{e}\right)$ admits a harmonic map with constant energy in a sphere (4]). Existence of maximal metrics for the aforementioned functional, which are a particular case of extremal metrics, have only been proved in two dimensions $(9,10,11,12])$. We would like to point out that a map $U:\left(M^{n}, g\right) \rightarrow\left(\mathbb{S}^{p-1}, g_{r}\right)$ is harmonic if $U=\left(u_{1}, \cdots, u_{p}\right)$ and each coordinate function is an eigenfunction associated to the same eigenvalue $\lambda_{k}\left(-\Delta_{g}\right)$.

Proof of Theorem 1.1. Let us denote by $E_{k}\left(L_{g_{e}}\right)$ the eigenspace corresponding to the $k$-th eigenvalue $\lambda_{k}\left(L_{g_{e}}\right)$, and consider the subset $K \subset L^{2}\left(M^{n}, g_{e}\right)$ defined by

$$
K:=\left\{u^{2} \mu_{e}^{2}: u \in E_{k}\left(L_{g_{e}}\right) \text { and }\|u\|_{L^{2}\left(M, g_{e}\right)}^{2}=1\right\} .
$$

This subset is compact and lies in a finite dimensional subset of $L^{2}\left(M, g_{e}\right)$. By Caratheodory's Theorem for Convex Hulls one deduce that the convex hull of $K$,

$$
\operatorname{Conv}(K)=\left\{\sum_{\text {finite }} a_{j} u_{j}^{2} \mu_{e}^{2}: a_{j} \geq 0, \sum a_{j}=1, u_{j} \in E_{k}\left(L_{g_{e}}\right),\left\|u_{j}\right\|_{L^{2}\left(M, g_{e}\right)}^{2}=1\right\},
$$

is compact as well. 
The first step of the proof is to show that $1 \in \operatorname{Conv}(K)$. On the contrary, let us assume that $\{1\}$ and $\operatorname{Conv}(K)$ have empty intersection. Since they are both convex and nonempty, the former one closed and the latter one compact, Hahn Banach Separation Theorem gives us the existence of a functional $\Phi \in\left(L^{2}\left(M, g_{e}\right)\right)^{*}$ separating $\{1\}$ from $\operatorname{Conv}(K)$ :

$$
\Phi(1)>0
$$

and

$$
\Phi(\varphi) \leq 0, \quad \forall \varphi \in \operatorname{Conv}(K)
$$

Furthermore, Riesz's Representation Theorem provides us with the existence of a function $f \in L^{2}\left(M, g_{e}\right)$ such that $\Phi$ is defined by integrating against $f d v_{g_{e}}$. This allow us to rewrite (3.15) and (3.16) as

$$
\int_{M} f d v_{g_{e}}>0
$$

and

$$
\int_{M} \varphi f d v_{g_{e}} \leq 0, \forall \varphi \in \operatorname{Conv}(K)
$$

We let $w=f-\int_{M} f d v_{g_{e}}$ be the zero mean value part of $f$, and consider the analytic deformation $g(t)$ of $g_{e}$ generated by the function $\mu_{t}=e^{t w \mu_{e}^{2}}$. As before, we use $h$ to denote $\left.\frac{d}{d t} \mu_{t}\right|_{t=0}=w \mu_{e}^{2}$. From (3.17) and (3.18) one gets that for all $u \in E_{k}\left(L_{g_{e}}\right)$,

$$
\int_{M} h u^{2} d v_{g_{e}}=\underbrace{\int_{M} u^{2} \mu_{e}^{2} f d v_{g_{e}}}_{\leq 0}-\underbrace{\left(\int_{M} f d v_{g_{e}}\right)}_{>0} \underbrace{\left(\int_{M} u^{2} \mu_{e}^{2} d v_{g_{e}}\right)}_{>0} .
$$

This implies that the quadratic form

$$
-\frac{4}{n-2} \lambda_{k}\left(L_{g_{e}}\right) \int_{M} h u^{2} d v_{g_{e}}
$$

has a constant sign on $E_{k}\left(L_{g_{e}}\right)$.

Let $m$ be the multiplicity of $\lambda_{k}\left(L_{g_{e}}\right)$, and denote by $\left\{u_{i}\right\}_{i=1}^{m}$ the orthonormal basis arising from the generating function $\mu_{t}$ as in Theorems 2.1 and 2.2. Then

$$
\Lambda_{i}^{\prime}(0)=-\frac{4}{n-2} \lambda_{k}\left(L_{g_{e}}\right) \int_{M} h u_{i}^{2} d v_{g_{e}}
$$

has constant sign for all $i=1, \cdots, m$. By means of Corollary 3.1 we deduce that the one-sided derivatives of $F_{g}^{k}\left(\mu_{t}^{4 /(n-2)} g_{e}\right)$ at $t=0$ are both of the same sign. This contradicts the extremality assumption on $g_{e}$. Hence, $1 \in \operatorname{Conv}(K)$, i.e. there exists a finite collection $u_{1}, \cdots, u_{p} \in E_{k}\left(L_{g_{e}}\right)$ such that $\sum_{i=1}^{p} u_{i}^{2} \mu_{e}^{2} \equiv 1$.

We now show that the equation (1.13) holds. To this end, we apply the LaplaceBeltrami operator to the relation $\sum_{i=1}^{p} u_{i}^{2} \equiv \mu_{e}^{-2}$ : 


$$
\begin{aligned}
-\frac{1}{2} \Delta_{g_{e}}\left(\mu_{e}^{-2}\right) & =-\frac{1}{2} \Delta_{g_{e}}\left(\sum_{i=1}^{p} u_{i}^{2}\right)=-\sum_{i=1}^{p}\left(u_{i} \Delta_{g_{e}} u_{i}+\left|\nabla_{g_{e}} u_{i}\right|_{g_{e}}^{2}\right) \\
& =\sum_{i=1}^{p}\left(u_{i} L_{g_{e}} u_{i}-\left|\nabla_{g_{e}} u_{i}\right|_{g_{e}}^{2}-c_{n} R_{g_{e}} u_{i}^{2}\right) \\
& =\lambda_{k}\left(L_{g_{e}}\right) \mu_{e}^{-2}-\sum_{i=1}^{p}\left|\nabla_{g_{e}} u_{i}\right|_{g_{e}}^{2}-c_{n} R_{g_{e}} \mu_{e}^{-2} .
\end{aligned}
$$

The result is obtained after solving for $\lambda_{k}\left(L_{g_{e}}\right)$.

Finally, if the extremal metric $g_{e}$ coincides with the background metric $g$, then $\mu_{e}=1$. Therefore, from (1.13),

$$
\lambda_{k}\left(L_{g}\right)=c_{n} R_{g}+\sum_{i=1}^{p}\left|\nabla_{g} u_{i}\right|^{2} .
$$

This gives us

$$
\begin{aligned}
L_{g} u_{j}=\lambda_{k}\left(L_{g}\right) u_{j} & \Longleftrightarrow\left(-\Delta_{g}+c_{n} R_{g}\right) u_{j}=\left(c_{n} R_{g}+\sum_{i=1}^{p}\left|\nabla_{g} u_{i}\right|^{2}\right) u_{j} \\
& \Longleftrightarrow-\Delta_{g} u_{j}=\left(\sum_{i=1}^{p}\left|\nabla_{g} u_{i}\right|^{2}\right) u_{j},
\end{aligned}
$$

which is the harmonic map equation for maps into spheres ([7]). This finishes the proof.

We remark that equation (3.23) implies

$$
\lambda_{k}\left(L_{g}\right) \geq c_{n} R_{g}
$$

Therefore, if $\lambda_{k}\left(L_{g}\right)<0$, then $R_{g}<0$ everywhere on $M^{n}$.

3.1. $\mathbf{\Lambda}_{\mathbf{1}}(\mathbf{M}, \mathbf{g})$ for the case $\mathbf{Y}(\mathbf{M},[\mathbf{g}])>\mathbf{0}$. We are given a closed n-dimensional Riemannian manifold $M$ endowed with a conformal class $[g]$ satisfying $Y(M,[g])>$ 0 . We focus now on $\lambda_{1}\left(L_{\tilde{g}}\right)$, where $\tilde{g} \in[g]$. It is well known that the multiplicity of $\lambda_{1}\left(L_{\tilde{g}}\right)$ is always 1 .

Proposition 1.2 implies that the maximization problem "finding $\tilde{g} \in[g]$ such that $F_{g}^{1}(\tilde{g})=\Lambda_{1}(M, g)$ " would not have a solution if the scalar curvature $R_{g}$ with respect to the reference metric $g$ does not satisfies $R_{g}>0$ everywhere on $M^{n}$. That is, $R_{g}>0$ is necessary. Finding such a reference metric within a conformal class is always possible as long as $Y(M,[g])>0$, which we are assuming.

Proof. Since $\lambda_{1}\left(L_{g_{e}}\right)$ has multiplicity one, it satisfies $\lambda_{1}\left(L_{g_{e}}\right)<\lambda_{2}\left(L_{g_{e}}\right)$, and so Theorem 1.1] gives us that $u^{2}=\mu_{e}^{-2}$, where $u$ is an eigenfunction associated to $\lambda_{1}\left(L_{g_{e}}\right)$. Therefore $u= \pm \mu_{e}^{-1}$, and

$$
\lambda_{1}\left(L_{g_{e}}\right)\left( \pm \mu_{e}^{-1}\right)=\lambda_{1}\left(L_{g_{e}}\right) u=L_{g_{e}}(u)=L_{g_{e}}\left( \pm \mu_{e}^{-1}\right) .
$$

Using the transformation law (1.2), we get

$$
\lambda_{1}\left(L_{g_{e}}\right)\left(\mu_{e}^{-1}\right)=\mu_{e}^{-\frac{n+2}{n-2}} L_{g}\left(\mu_{e} \mu_{e}^{-1}\right)=\mu_{e}^{-\frac{n+2}{n-2}} L_{g}(1)=\mu_{e}^{-\frac{n+2}{n-2}}\left(c_{n} R_{g}\right)
$$

The result is obtained after solving for $c_{n} R_{g}$. 
We remark that Proposition 1.2 follows directly from Corollary 3.1 and the simplicity of $\lambda_{1}\left(L_{g_{e}}\right)$. Indeed, since $E_{1}\left(L_{g_{e}}\right)$ is generated by one function, say $u$, formulas (3.6) and (3.7) are equal to each other. That is, the functional $F_{g}^{1}$ is differentiable at $t=0$ along any analytic deformation $g(t)$ of $g_{e}$. Then the extremal condition on $g_{e}$ implies that for any $h \in C^{\infty}(M)$,

$$
\frac{4}{n-2} \lambda_{1}\left(L_{g_{e}}\right)\left\{\int_{M} h \mu_{e}^{\frac{4}{n-2}} d v_{g}-\int_{M} h u^{2} d v_{g_{e}}\right\}=0 .
$$

This is equivalent to

$$
\int_{M} h\left(\mu_{e}^{-2}-u^{2}\right) d v_{g_{e}}=0 .
$$

Since $h$ is arbitrary, we deduce that $\mu_{e}^{-2}=u^{2}$, and the result follows in a similar manner.

In terms of maximal metrics, the condition $R_{g}>0$ everywhere on $M^{n}$ is also sufficient for existence of conformal metrics achieving $\Lambda_{1}\left(M^{n}, g\right)$. This is the result stated in Theorem 1.3.

Proof of Theorem 1.3. Let us start by noticing that $\int_{M} \mu_{\max }^{4 /(n-2)} d v_{g}=1$. We first show that $c_{n} \int_{M} R_{g} d v_{g}$ is an eigenvalue for $L_{g_{\max }}$ with eigenfunction $\mu_{\max }^{-1}$, and, moreover, $\lambda_{1}\left(L_{g_{\max }}\right)=c_{n} \int_{M} R_{g} d v_{g}$. This is a consequence of the conformal properties of the conformal laplacian:

$$
\begin{aligned}
L_{g_{\max }}\left(\mu_{\max }^{-1}\right) & =\mu_{\max }^{-\frac{n+2}{n-2}} L_{g}(1)=\mu_{\max }^{-\frac{n+2}{n-2}} c_{n} R_{g} \\
& =\mu_{\max }^{-\frac{n+2}{n-2}} c_{n} \cdot \mu_{\max }^{\frac{4}{n-2}} \int_{M} R_{g} d v_{g}=\left(c_{n} \int_{M} R_{g} d v_{g}\right) \mu_{\max }^{-1} .
\end{aligned}
$$

To argue that $c_{n} \int_{M} R_{g} d v_{g}$ is, in fact, the smallest eigenvalue of $L_{g_{\max }}$, i.e. $\lambda_{1}\left(L_{g_{\max }}\right)=$ $c_{n} \int_{M} R_{g} d v_{g}$, we observe that the eigenfunction $\mu_{\max }^{-1}$ has constant sign.

It remains to show that $F_{g}^{1}\left(g_{\max }\right)=\Lambda_{1}(M, g)$. To this end, pick an arbitrary metric $\tilde{g}=\tilde{\mu}^{4 /(n-2)} g \in[g]$. By the variational characterization of $\lambda_{1}\left(L_{\tilde{g}}\right)$ with $u=\tilde{\mu}^{-1}$ as test function, we get

$$
\begin{aligned}
\lambda_{1}\left(L_{\tilde{g}}\right) \cdot \int_{M} \tilde{\mu}^{\frac{4}{n-2}} d v_{g} & \leq \frac{\int_{M} \tilde{\mu}^{-1} L_{\tilde{g}}\left(\tilde{\mu}^{-1}\right) d v_{\tilde{g}}}{\int_{M} \tilde{\mu}^{-2} d v_{\tilde{g}}} \cdot \int_{M} \tilde{\mu}^{\frac{4}{n-2}} d v_{g} \\
& =\frac{\int_{M} \tilde{\mu}^{-1} \cdot \tilde{\mu} L_{g}\left(\tilde{\mu} \cdot \tilde{\mu}^{-1}\right) d v_{g}}{\int_{M} \tilde{\mu}^{-2} \tilde{\mu}^{\frac{2 n}{n-2}} d v_{g}} \cdot \int_{M} \tilde{\mu}^{\frac{4}{n-2}} d v_{g} \\
& =\frac{\int_{M} L_{g}(1) d v_{g}}{\int_{M} \tilde{\mu}^{\frac{4}{n-2}} d v_{g}} \cdot \int_{M} \tilde{\mu}^{\frac{4}{n-2}} d v_{g}=c_{n} \int_{M} R_{g} d v_{g}=\lambda_{1}\left(L_{g_{\max }}\right) .
\end{aligned}
$$

This finishes the proof. 
As a particular case of the previous theorem, we get Sire-Xu Theorem on the existence of maximal metrics for $\Lambda_{1}\left(\mathbb{S}^{n}, g_{r}\right)$, where $g_{r}$ is the standard round metric on the sphere $\mathbb{S}^{n}$.

Theorem 3.3 (Sire-Xu). For the standard round sphere $\left(\mathbb{S}^{n}, g_{r}\right)$, the only maximizer for $\Lambda_{1}\left(\mathbb{S}^{n}, g_{r}\right)$, up to scalings, is the standard round metric $g_{r}$.

Proof. It is well known that $R_{g_{r}}=n(n-1)$. From Theorem 1.3 we get that $g_{\max }=\omega_{n}^{-1} g_{r}$ solves the maximization problem for $F_{g_{r}}^{1}$. Here $\omega_{n}$ denotes the volume of $\mathbb{S}^{n}$ with respect to $g_{r}$. If $\tilde{g}=\tilde{\mu}^{4 /(n-2)} g_{r} \in\left[g_{r}\right]$ maximizes $F_{g_{r}}^{1}$, i.e. if $F_{g_{r}}^{1}(\tilde{g})=\Lambda_{1}\left(\mathbb{S}^{n}, g_{r}\right)$, then it has to satisfies equation (1.14). This implies that the conformal factor $\tilde{g}$ is a constant function. The proof is now completed.

We end this section with a comment on the uniqueness of maximal metrics for $\lambda_{1}(L$.$) . If we have a maximal metric for F_{g}^{1}$ and the scalar curvature of the reference metric $g$ is constant, then the conformal factor of the maximal metric is constant due to (1.14). This is the scenario in Theorem 3.3 .

3.2. $\boldsymbol{\Lambda}_{\mathbf{1}}(\mathbf{M}, \mathbf{g})$ for the case $\mathbf{Y}\left(\mathbf{M}^{\mathbf{n}},[\mathbf{g}]\right)<\mathbf{0}$. In this case, $\lambda_{1}\left(L_{\tilde{g}}\right)$ is strictly negative for any $\tilde{g} \in[g]$, and its multiplicity is still 1. Formula (1.14) in Proposition 1.2 remains valid in this case. For the sake of completeness, we state the result but omit the proof as it is the same argument.

Corollary 3.4. Suppose $[g]$ is a conformal class for which $Y\left(M^{n},[g]\right)<0$, and assume that $g_{e}=\mu_{e}^{4 /(n-2)} g$ is an extremal metric for $F_{g}^{1}$ with $\int_{M} \mu_{e}^{4 /(n-2)} d v_{g}=1$. Then

$$
c_{n} R_{g}=\lambda_{1}\left(L_{g_{e}}\right) \mu_{e}^{\frac{4}{n-2}} .
$$

In particular, the scalar curvature $R_{g}$ with respect to the background metric $g$ is negative everywhere.

As before, this put a restriction on the choice of background metric. In order to solve the maximization problem for $\Lambda_{1}(M, g)$ in this case, $R_{g}<0$ is a necessary condition. The proof of the following theorem is exactly as that of Theorem 1.3 , and thus the majority of the arguments are omitted.

Theorem 3.5. Let $M$ be a closed n-dimensional manifold endowed with a conformal class $[g]$ satisfying $Y\left(M^{n},[g]\right)<0$. If $R_{g}<0$, then the metric

$$
g_{\max }:=\mu_{\max }^{\frac{4}{n-2}} g=\left(\frac{R_{g}}{\int_{M} R_{g} d v_{g}}\right) g
$$

solves the maximization problem for $\Lambda_{1}(M, g)$, i.e. $F_{g}^{1}\left(g_{\max }\right)=\Lambda_{1}(M, g)$. In particular, $\Lambda_{1}(M, g)<0$.

Proof. Note that since $R_{g}$ is negative everywhere the conformal factor $\mu_{\max }$ is well defined. As in Theorem [1.3. we get $F_{g}^{1}\left(g_{\max }\right)=c_{n} \int_{M} R_{g} d v_{g}=\Lambda_{1}(M, g)$, and therefore $\Lambda_{1}(M, g)$ is strictly negative.

\section{REFERENCES}

[1] B. Ammann and P. Jammes, The supremum of conformally covariant eigenvalues in a conformal class, in Variational Problems in Differential Geometry, London Mathematical Society Lecture Note Series, vol. 394, Cambridge (2011), 1-23. 
[2] S. Bando and H. Urakawa, Generic properties of the eigenvalue of Laplacian for com- pact Riemannian manifolds, Tôhoku Math. J., 35 (1983), 155-172.

[3] Y. Canzani, On the multiplicity of eigenvalues of conformally covariant operators, Annales de I'Institut Fourier, 2014.

[4] A. El Soufi and S. Ilias, Laplacian eigenvalue functionals and metric deformations on compact manifolds, Journal of Geometry and Physics. 58 (2008), no.1, 89-104.

[5] A. El Soufi and S. Ilias, Riemannian manifolds admitting isometric immersions by their first eigenfunctions, Pacific Journal Of Mathematics, 195 (2000), 91-99.

[6] M.J. Gursky and S. Pérez-Ayala, Variational properties of the second eigenvalue of the Conformal Laplacian, arXiv:2010.13210 [math.DG], 2020.

[7] F. Hélein and J.C. Woods, Harmonic Maps, in Handbook of Global Analysis, Elsevier (2008), p. $417-491$.

[8] J. Kazdan and F. Warner, Scalar curvature and conformal deformations of Riemannian structure, Journal of Differential Geometry 10 (1975), 113-134.

[9] N. Nadirashvili and Y. Sire, Conformal spectrum and harmonic maps, Moscow Mathematical Journal, 15(1):123-140, 2015.

[10] N. Nadirashvili and Y. Sire, Maximization of higher order eigenvalues and applications, Moscow Mathematical Journal, 15(4):767-775, 2015.

[11] R. Petrides, Existence and regularity of maximal metrics for the first Laplace eigenvalue on surfaces, Geometric and Functional Analysis 24(4):1336-1376, 2014.

[12] R. Petrides, On the existence of metrics that maximize Laplace eigenvalue on surfaces, International Mathematics Research Notices, 2018(14): 4261-4355, 2018

[13] Y. Sire and H. Xu, Upper bounds for eigenvalues of conformal laplacian on spheres, arXiv:1809.06874v1 [math.DG] (2018)

Department of Mathematics, University of Notre Dame, Notre Dame, IN 46556

Email address: sperezay@nd.edu 